\title{
MILLIMETER WAVE ABSORPTION IN HYDROXYAPATITE AND 3YSZ CERAMICS IN WIDE TEMPERATURE RANGE
}

\author{
A. Eremeev, S. Egorov, V. Kholoptsev \\ Institute of Applied Physics, Russian Academy of Sciences, 46 Ulyanov str., Nizhny \\ Novgorod, 603950, Russia \\ aeremeev@appl.sci-nnov.ru
}

Keywords: millimeter wave, absorption, ceramics

\section{Introduction}

In the field of ceramic-based materials processing the last three decades has been marked by significant academic and industry interest in Additive Manufacturing (AM) technology due to its capability to produce ceramic parts with complex geometry and customizable materials properties. Conceptually, AM technology is a layer-by-layer fabrication of three dimensional physical parts directly from computer-aided design [1]. Solidification of the parts prepared from substances containing ceramic powder may be performed either by conventional heat treatment of a part as whole or by directed energy deposition. Both these strategies can be implemented using gyrotron-based millimeter-wave facilities allowing alternatively both the uniform heating of large-size parts in multi-mode cavities and local heating by focused wave-beams [2].

AM technology proves a growing potential in biomedicine. Hydroxyapatite and yttriastabilized zirconia-based ceramics are widely used in biomedical applications due to their high biocompatibility. The knowledge of their microwave (MW) absorption variation with temperature and porosity as the materials are densified, is necessary to optimize the scheme of microwave heating.

Results of microwave absortption measurements in powder compacts of hydroxyapatite and yttria-stabilized zirconia are presented in this paper.

\section{Experimental procedure}

The samples in the shape of disks $8 \mathrm{~mm}$ in diameter and about $2.5 \mathrm{~mm}$ in thickness were compacted by uniaxial pressing under a pressure of $200 \mathrm{MPa}$ from commercially available hydroxyapatite (HA) GAP-85d (Polistom, Russia), and yttria-stabilized zirconia (3YSZ) powder (Tosoh corp., Japan). Phase composition of 3 YSZ was $12.5 \%$ of monoclinic and $87.5 \%$ of tetragonal phase. Green densities of the samples were $1.56 \mathrm{~g} / \mathrm{cm}^{3}(49.3 \%$ of theoretical value) for HA and $2.86 \mathrm{~g} / \mathrm{cm}^{3}$ (47.3\% of theoretical value) for $3 \mathrm{YSZ}$.

The measurements were performed at $24 \mathrm{GHz} 3 \mathrm{~kW}$ gyrotron system [2, 3]. For comparison the measurements of complex dielectric permittivity of hydroxyapatite at frequency of 180 $\mathrm{GHz}$ were made by spectrometer based on a high-Q $\left(\mathrm{Q}_{0} \sim 10^{6}\right)$ open Fabry-Perot resonator [4]. Phase composition of the samples was analysed with X-ray difractometer Rigaku Ultima IV.

Samples for measurements were placed into the gyrotron system applicator and surrounded with low loss porous alumina based thermal insulation (AL-30, ZIRCAR Ceramics, USA). Some experiments with preliminary sintered samples were performed without thermal insulation.

Schematic of the applicator and the sample position in it are shown in Fig.1. Sample temperature was measured with B-type thermocouple. Applicator door was equipped with a water cooled optical window made as a double quartz glass with flowing water inside. 

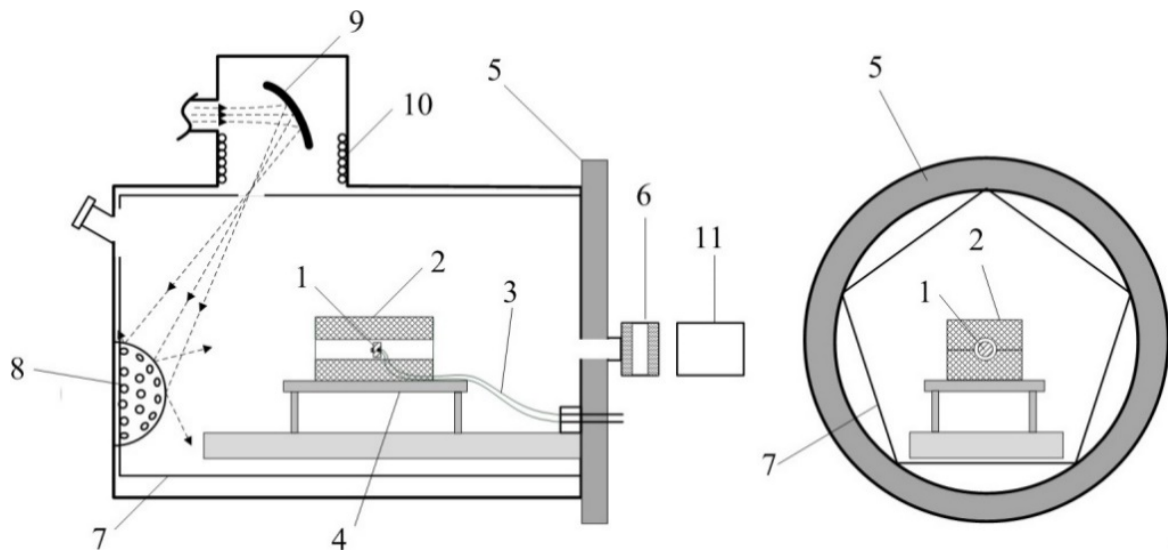

Fig. 1. Schematic of Gyrotron System Applicator: specimen (1), thermal insulation (2), thermocouple (3), support table (4), applicator door (5), optical window (6), pentaprism

(7), mode stirrer (8), focusing mirror (9), MW absorber (10), infrared camera (11).

The thermal insulation had a through hole of $10 \mathrm{~mm}$ in diameter allowing observation of the sample with infrared CMOS camera 3iCube IC4133IR (New Electronic Technology, Germany). Transfer and recording to a computer, and the display on the monitor were carried out using the software Stream Pix 7 (NORPIX, Canada). Thus, the design of the applicator and insulation allowed performing optical measurements of both the sample size and temperature distribution over the sample surface.

Upper part of the applicator contains a metal mirror directing the microwave radiation into a main chamber of the applicator through a coupling hole of $50 \mathrm{~mm}$ in diameter, and a microwave absorber based on a set of Teflon tubes with flowing water which absorbs the scattered radiation. The power of the radiation absorbed in the upper chamber of the applicator is measured by using a system consisting of water temperature meters at the input and output of the absorber, as well as a calibration heater with known power.

The applicator is equipped with a mode stirrer, representing an excentrically rotating hemisphere with hemispherical hollows drilled in it. Pentaprism shape of the applicator main chamber inner surface along with rotation of the mode stirrer provides uniform microwave field distribution over the volume of the applicator.

Measurements were made by calorimetric method, when microwave power absorbed in the sample is determined basing on the differences of the heating/cooling rates and MW power densities at the moments of intentional abrupt change of the microwave power at different sample temperatures. The method allows to measure absorption coefficients in real time during the sintering process. An example of the hydroxyapatite sample heating is presented in Fig. 2. Required temperature-time dependence was loaded to the gyrotron system control computer before start of the heating process. This dependence was automatically maintained by the gyrotron system control unit but from time to time an operator manually abruptly reduced the value of high voltage applied to the gyrotron in order to lower MW power fed the applicator. After some time (about $20 \mathrm{~s}$ ) the operator returned the control unit to the automatic control regime, and the sample temperature is automatically increased up to prescribed value. Then the procedure was repeated at the next value of the sample temperature. 


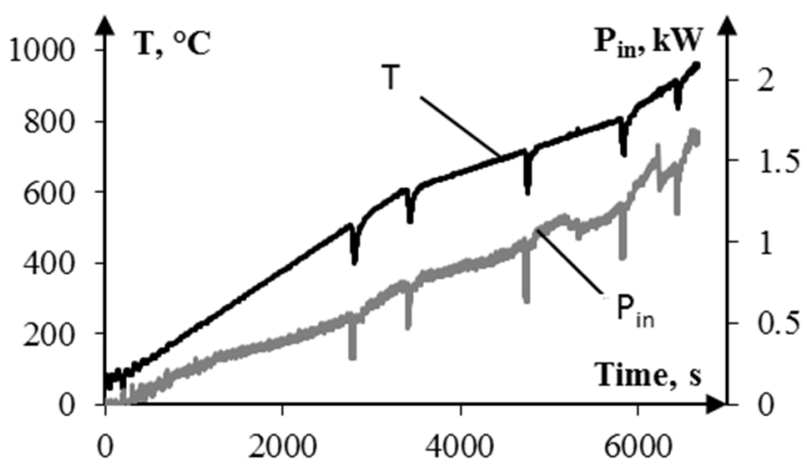

Fig. 2. Heating of hydroxyapatite sample: sample temperature (T), MW power fed the applicator $\left(\mathrm{P}_{\text {in }}\right)$.

\section{Results and discussion}

Energy balance equation is:

$C_{s} m\left[\frac{d T}{d t}\right]_{b(a)}=\alpha \Pi_{b(a)} S_{\text {sample }}-P_{\text {loss }}$,

where $C_{s}$ is specific heat capacity of the sample material, $\mathrm{m}$ is a mass of the sample, $\left[\frac{d T}{d t}\right]_{b(a)}$ is a heating/cooling rate, $\alpha$ is an absorption coefficient, $\Pi_{b(a)}$ is a MW power density incident to the sample, $\mathrm{S}_{\text {sample }}$ is a surface area of the sample, $\mathrm{P}_{\text {loss }}$ is a power in heat losses, subscripts $b$ and $a$ denote moments of time just before and just after the intentional change of MW power correspondingly.

Heat capacities $C_{s}$ as a functions of temperature were taken from [5] for HA, and from [6, 7] for 3 YSZ.

From (1) the absorption coefficient can be expressed as:

$\alpha=C_{s} m \frac{\left[\frac{d T}{d t}\right]_{b}-\left[\frac{d T}{d t}\right]_{a}}{\left(\Pi_{b}^{T E}-\Pi_{a}^{T E}\right) S_{\text {sample }}}=C_{s} m \frac{\left[\frac{d T}{d t}\right]_{b}-\left[\frac{d T}{d t}\right]_{a}}{\psi\left(\Pi_{b}^{a p p}-\Pi_{a}^{a p p}\right) S_{\text {sample }}}$

Here $\Pi_{b(a)}^{T E}$ are MW power densities inside the thermal insulation, $\Pi_{b(a)}^{a p p}$ are the power densities outside the thermal insulation, in the volume of the applicator, and $\psi$ is a ratio of MW power inside the thermal insulation to the power outside the thermal insulation:

$\psi=\frac{\Pi_{b(a)}^{T E}}{\Pi_{b(a)}^{a p p}}$

Power density in the volume of the applicator can be evaluated basing on its Q-factor:

$\Pi^{a p p}=\frac{c P_{\text {in }}}{\omega V_{\text {app }}} Q$

where $\mathrm{c}$ is the speed of light in free space, $\mathrm{P}_{\text {in }}$ is MW power fed the applicator, $\omega$ is the angular microwave frequency, $\mathrm{V}_{\text {app }}$ is the volume of the applicator, $\mathrm{Q}$ is the $\mathrm{Q}$-factor.

Q-factor for empty applicator can be calculated theoretically:

$\frac{1}{Q_{\text {empty }}}=\frac{1}{Q_{\text {ohm }}}+\frac{1}{Q_{c}}+\frac{1}{Q_{w}}$,

where $Q_{\text {ohm }}$ is ohmic Q-factor related to the absorption of microwave radiation in the applicator walls, $\mathrm{Q}_{\mathrm{c}}$ and $\mathrm{Q}_{\mathrm{w}}$ are coupling $\mathrm{Q}$-factors determining by the loss of MW radiation in the coupling hole and optical window correspondingly.

The Q-factors can be calculated by formulas [8]:

$Q_{c}=\frac{8 \pi V_{a p p}}{\lambda S_{c}}, \quad Q_{w}=\frac{8 \pi V_{a p p}}{\lambda S_{w}}, \quad Q_{o h m}=\frac{V_{a p p}}{s_{a p p} \delta_{s}}$, 
where $\lambda$ is a wavelength of MW radiation, $S_{c}$ and $S_{w}$ are the surface areas of the coupling hole and optical window correspondingly, $S_{\text {app }}$ is a surface area of the applicator, $\delta_{s}=c / \sqrt{2 \pi \omega \sigma}$ is a skin depth, $\sigma=1.49 \cdot 10^{7}(\mathrm{Ohm} \cdot \mathrm{m})^{-1}$ is the specific conductivity of the chamber wall material (aluminium alloy). Substituting the applicator parameters $\left(\mathrm{V}_{\text {app }}=5.7 \cdot 10^{4} \mathrm{~cm}^{3}, \mathrm{~S}_{\text {app }}=9 \cdot 10^{3} \mathrm{~cm}^{2}\right)$ one can obtain:

$Q_{\text {empty }}=2.5 \cdot 10^{4}$

Q-factor of the applicator can be estimated in real time during the heating process using the approach described in [3]. According to the definition of the Q-factor:

$Q_{i}=\frac{W \omega}{P_{i}}$,

where $\mathrm{Q}_{\mathrm{i}}$ is the $\mathrm{Q}$-factor of the $i$-th loss channel, $\mathrm{W}$ is the electromagnetic energy stored in the applicator volume, and $\mathrm{P}_{\mathrm{i}}$ is the power going to the $i$-th loss channel, one can conclude: $Q=Q_{c} \frac{P_{c}}{P_{\text {in }}}$,

where $\mathrm{P}_{\mathrm{c}}$ is $\mathrm{MW}$ power lost in the coupling hole, $\mathrm{P}_{\mathrm{in}}$ is a microwave power injected to the applicator.

As the upper part of the applicator is equipped with effective MW power absorber along with the system for measuring the absorbed power, the value of $\mathrm{P}_{c}$ was measured in real time during each experiment. The value of the power injected to the applicator $\mathrm{P}_{\text {in }}$ was determined from the gyrotron operating parameters basing on preliminary calibration experiments. Obtained by this approach Q-factor for empty furnace was $\mathrm{Q}_{\text {empty }}=(2.2 \pm 0.1) \cdot 10^{4}$, which is in very good agreement with the theoretical value (7). Typical dependence of applicator Qfactors on the sample temperature, when the applicator is loaded with both the sample and the thermal insulation is shown in Fig. 3 (a). It is seen that the values of Q-factor of the loaded applicator are close to the Q-factor of empty applicator.

After the Q-factor of the applicator is known, MW power density in the volume of the applicator can be found according to (4), but as it was mentioned above, MW power density inside the thermal insulation could differ from the power density outside the thermal insulation.

The following procedure was used to evaluate MW power density inside the thermal insulation. At the first stage, the samples of both composites were sintered in gyrotron system using described above thermal insulation. At the second stage the sintered samples were placed in the thermal insulation and heated in the regimes similar to shown in Fig. 2. At the last, third stage, the same samples were heated without thermal insulation, when the sample was fixed in open space with a purposely bent thermocouple wires. To reach high temperatures the last heating was made in vacuum, with a residual pressure of $15 \mathrm{~Pa}$. When heating without thermal insulation the values of absorptivity of the sintered samples $\alpha_{s}(T)$ can be defined from formula (2) with $\psi=1$.

Knowing this absoprtivity allows to evaluate the value of $\psi$ from (2), when heating the same sintered sample in the thermal insulation:

$\psi=C_{s} m \frac{\left[\frac{d T}{d t}\right]_{b}-\left[\frac{d T}{d t}\right]_{a}}{\alpha_{s}(T) \cdot\left(\Pi_{b}^{a p p}-\Pi_{a}^{a p p}\right) S_{\text {sample }}}$,

where $\alpha_{s}(T)$ is a value of absorption coefficient of the sintered sample, which was defined from experiments without thermal insulation.

The dependence of $\psi$ on the sample temperature is presented in Fig. 3 (b) for both compositions: hydroxyapatite (HA) and yttria-stabilized zirconia (3YSZ).

Absorptivity of initially raw samples were evaluated basing on (2) with ratio $\psi$ defined above. During heating the samples shrunk because of sintering process and the value of the sample surface area $\mathrm{S}_{\text {sample in }}(2)$ decreased. 


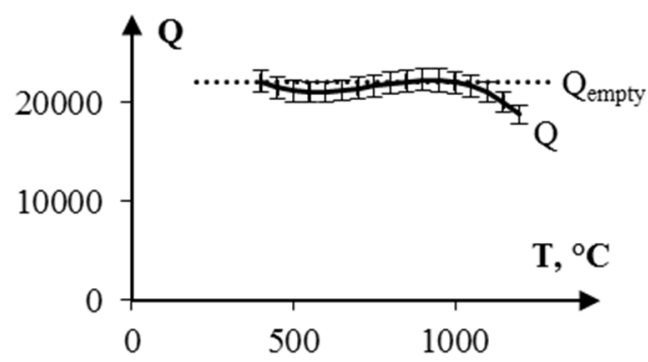

(a)

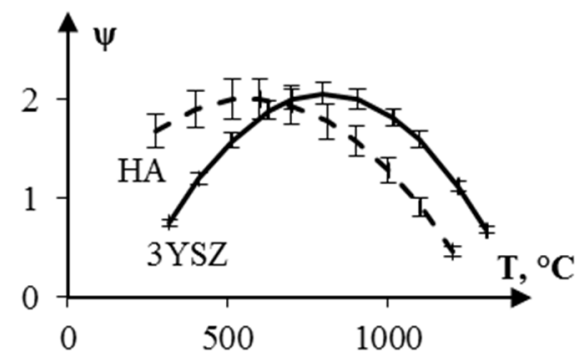

(b)

Fig. 3. Q-factor of the applicator (a) and ratio $\psi\left(\right.$ b) versus sample temperature, $Q_{\text {empty }}$ is Qfactor of empty applicator.

Basing on optical images from CMOS camera the sample diameter was measured in real time, allowing substitution of the real time values of $S_{\text {sample }}$ to (2).

Finally, as all parameters in (2) have been defined, the absorptivities of raw samples of the both compositions were obtained. The results along with the absorptivities of sintered samples are presented in Fig. 4.

Calculated above absorptivities depend not only on the materials properties, but also on the shape and dimensions of the samples. To reveal the intrinsic properties of the materials one should know the electric field inside the sample. For the samples with dimensions on the order of the wavelength and at isotropic irradiation as a rough estimation the electric field in the volume of the sample can be considered approximately equal to the value of electric field outside the sample. In this case:

$\alpha \Pi S_{\text {sample }}=\frac{\omega}{8 \pi} \varepsilon^{\prime \prime}|E|^{2} V_{\text {sample }}$,

where $\Pi=\frac{c}{8 \pi}|E|^{2}$ is MW power density, $\varepsilon^{\prime \prime}$ is an imaginary part of the complex permittivity, $\mathrm{E}$ is an electric field of the electromagnetic wave.

From (11) it is easy to get:

$\varepsilon^{\prime \prime}=\frac{c}{\omega h_{\text {sample }}} \alpha$,

where $h_{\text {sample }}$ is a thickness of the sample. Note that $h_{\text {sample }}$ decreases during the sintering process of raw samples.

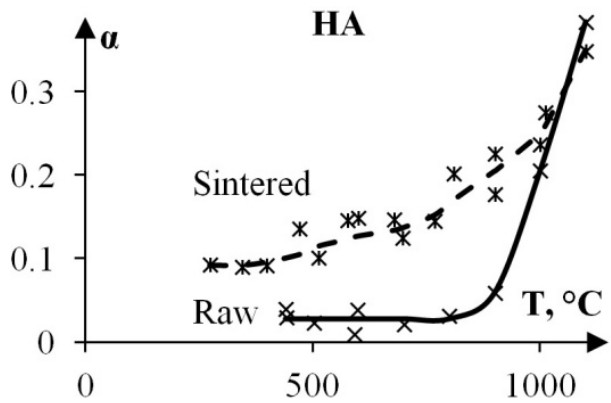

(a)

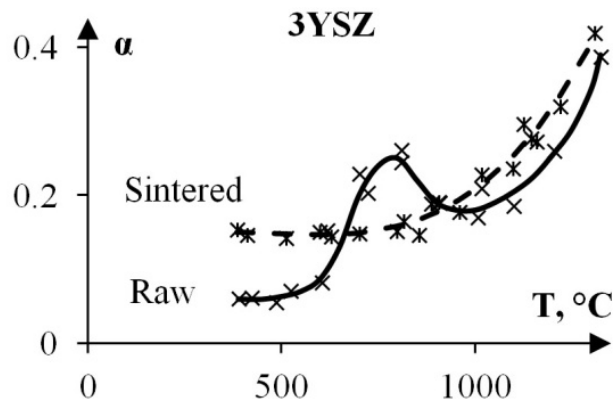

(b)

Fig. 4. Samples absorptivity: HA (a), 3YSZ (b), raw samples (solid lines), sintered samples (dashed lines). 


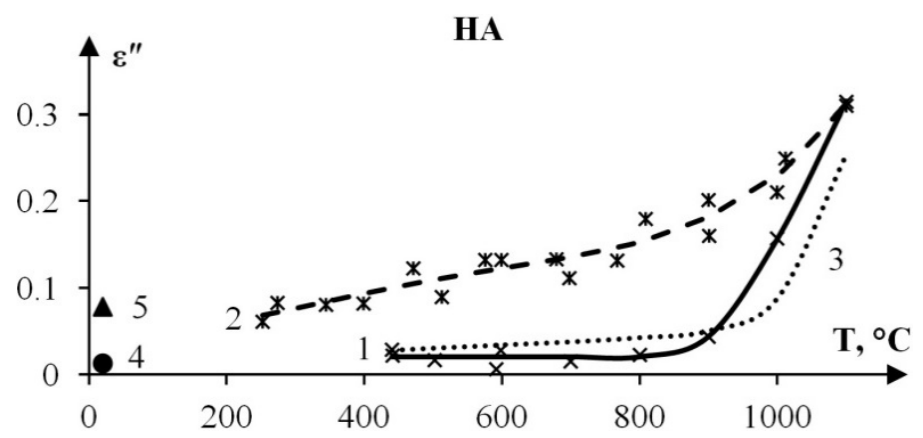

Fig. 5. Imaginary part of hydroxyapatite permittivity: raw samples (1), sintered samples (2), Effective Medium Approximation (3), raw sample at $180 \mathrm{GHz}$ (4), $180 \mathrm{GHz}$ sintered sample at $180 \mathrm{GHz}(5)$.

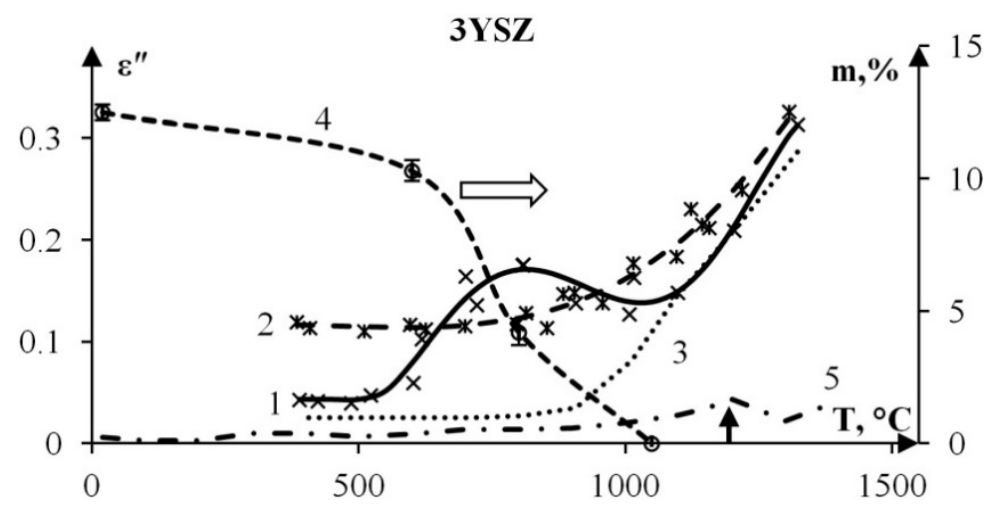

Fig. 6. Imaginary part of 3 YSZ permittivity: raw samples (1), sintered samples (2),

Effective Medium Approximation (3), content of monoclinic phase (4), pure zirconia powder at $3.8 \mathrm{GHz}$ [11] (5), solid arrow shows m-t transformation area for pure zirconia.

Figs. 5, 6 show the results of calculations of $\varepsilon^{\prime \prime}$ using formula (12) and taking into account shrinkage of the samples.

Measurements of the complex permittivity of HA sintered in conventional furnace by FabryPerot technique [4] at room temperature gave the following results:

$\varepsilon^{\prime}=2.82, \varepsilon^{\prime \prime}=0.013 \pm 0.001$ (raw sample);

$\varepsilon^{\prime}=9.42, \varepsilon^{\prime \prime}=0.078 \pm 0.008$ (sintered sample).

These values are included to Fig. 5. It is seen that the values for $180 \mathrm{GHz}$ and $24 \mathrm{GHz}$ are in good agreement with each other.

In [9] the results of DC conductivity measurements of zirconia - yttria sintered compositions heating by MW radiation at frequency of $24 \mathrm{GHz}$ are presented. It was found that DC conductivity depends strongly on the thermal insulation material in which the sample was placed. At temperature $\mathrm{T}=500{ }^{\circ} \mathrm{C}$ for composition 3YSZ DC conductivity of the sample placed into 2YSZ container was $0.093(\mathrm{Ohm} \cdot \mathrm{m})^{-1}$, and in the case when the sample was placed into alumina container the conductivity was $0.22(\mathrm{Ohm} \cdot \mathrm{m})^{-1}$. Calculation of high frequency conductivity from our data $\left(\sigma=\frac{\omega}{4 \pi} \varepsilon^{\prime \prime}\right)$ gives the value of $0.15(\mathrm{Ohm} \cdot \mathrm{m})^{-1}$, which is just in between the values of DC conductivity mentioned above. It means that the mechanisms of both DC and high frequency conductivities are similar. 
Imaginary part of the permittivity for HA and 3YSZ raw samples show significantly different behaviour with temperature: while for HA $\varepsilon^{\prime \prime}$ smoothly increases with temperature, $\varepsilon^{\prime \prime}$ of 3 YSZ has a peak with its maximum at $800^{\circ} \mathrm{C}$.

According to X-ray analysis observed peak in $\varepsilon^{\prime \prime}$ corresponds to transition from monoclinic (m) to tetragonal $(\mathrm{t})$ phase, which started at $600{ }^{\circ} \mathrm{C}$ and finished at less than $1000{ }^{\circ} \mathrm{C}$ (see Fig. 8). After $1000{ }^{\circ} \mathrm{C}$ only tetragonal phase was found. These data are in accordance with [10], where the detailed phase diagram of zirconia - yttria system was presented. For the composition of zrconia with $3 \mathrm{~mol} \%$ yttria $\mathrm{m}$ - $\mathrm{t}$ transition occurred in the temperature interval from $560{ }^{\circ} \mathrm{C}$ to $800{ }^{\circ} \mathrm{C}$ [10]. Note that for pure zirconia this transition takes place at temperature of $1200{ }^{\circ} \mathrm{C}$.

It is known that $\mathrm{m}-\mathrm{t}$ transition in pure zirconia causes nonmonotonic temperature dependence of the $\varepsilon^{\prime \prime}$ at high frequencies [11]. Fig. 6 presents data from [11], illustrating peak in $\varepsilon^{\prime \prime}$ during monoclinic - tetragonal transition at frequency of $3.8 \mathrm{GHz}$ for loose zirconia powder (density $22 \%$ of theoretical value). Taking into account this behaviour and shift of $\mathrm{m}-\mathrm{t}$ transition to lower temperatures for zirconia - yttria composition it is reasonable to suppose that observed in our experiments peak in $\varepsilon^{\prime \prime}$ is a result of $\mathrm{m}-\mathrm{t}$ transformation.

Difference between imaginary part of the permittivity of raw and sintered samples for both compositions clearly seen in Figs. 5, 6 can be explained by their different porosity. To prove it a series of calculations of the materials permittivities based on Effective Medium Approximation (EMA) [12] were performed. According to EMA approach effective dielectric permittivity $\varepsilon_{\mathrm{e}}=\varepsilon_{\mathrm{e}}{ }^{\prime}+\mathrm{i} \varepsilon_{\mathrm{e}}{ }^{\prime \prime}$ of the porous media satisfies the equation:

$\sum_{i=1}^{n} p_{i} \frac{\varepsilon_{i}-\varepsilon_{e}}{\varepsilon_{i}-2 \varepsilon_{e}}=0$,

where $\mathrm{p}_{\mathrm{i}}$ and $\varepsilon_{\mathrm{i}}=\varepsilon_{\mathrm{i}}{ }^{\prime}+\mathrm{i} \varepsilon_{\mathrm{i}}{ }^{\prime \prime}$ are the volume ratio and complex dielectric permittivity of the $\mathrm{i}$-th component of the medium (including pores), correspondingly, $\mathrm{n}$ is a number of components. In the calculations obtained above values of imaginary parts $\varepsilon_{i}^{\prime \prime}$ of the both sintered materials were used. Real parts of the permittivity $\varepsilon_{\mathrm{i}}{ }^{\prime}$ were assumed independent on the temperature.

For HA a value $\varepsilon^{\prime}=9.42$ measured at frequency of $180 \mathrm{GHz}$ was used. As for 3 YSZ literature date for $\varepsilon^{\prime}$ were used. For monoclinic zirconia a big scatter of data in the range from 12 to 22 is presented in literature, for tetragonal sirconia the data are near $40[13,14]$. In our calculation the $\varepsilon^{\prime}=17.9$ for monoclinic phase and $\varepsilon^{\prime}=34.5$ for tetragonal phase [14] were used.

The results of calculations are presented in Figs. 5, 6. Calculated dependency of the imaginary part of the permittivity $\varepsilon^{\prime \prime}$ for hydroxyapatite is in good accordance with the experimental curve (see Fig. 5). As for 3 YSZ calculated value of $\varepsilon^{\prime \prime}$ smoothly increases with temperature, which doesn't correspond to the experimental data. Taking into account that in formula (13) $\varepsilon_{\mathrm{i}}^{\prime \prime}$ for the sintered material, which is completely in tetragonal phase, was substituted, the discrepancy between measured and calculated behaviour of $\varepsilon^{\prime \prime}$ can be explained by the nonmonotonic nature of zirconia permittivity imaginary part at $\mathrm{m}-\mathrm{t}$ transformation described above.

\section{Conclusion}

Absorptivities of both raw and sintered samples of hydroxyapatite and 3YSZ compositions at frequency of $24 \mathrm{GHz}$ in wide range of temperatures have been measured.

Imaginary parts of permittivities as functions of temperatures for both compositions were evaluated.

Nonmonotonic dependence of $3 \mathrm{YSZ} \varepsilon^{\prime \prime}$ on temperature caused possibly by monoclinic tetragonal phase transition in zirconia was found. 
Calculations of $\varepsilon^{\prime \prime}$ based on Effective Medium Approximation are in good agreement with experimental values for hydroxyapatite, but don't describe the peak in $\varepsilon^{\prime \prime}$ for 3 YSZ.

\section{Acknowledgement}

This research was supported by Russian Basic Research Foundation, grant \# 18-29-11045.

\section{References}

1. Vaezi, M., et al., Int. J. Adv. Manuf. Technol., 2013, 67, 1721-1759.

2. Bykov, Yu., et al., Radiophysics and Quantum Electronics, 2019, 61 (10) 752762.

3. Volkovskaya, I.I., et al., Radiophysics and Quantum Electronics, 2018, 61 (4), $286-295$.

4. V. V. Parshin, V.V., et al, Radiophysics and Quantum Electronics, 2009, 52 (8), 525-535

5. Egan, E.P., et al.., Journal of the American Chemical Society, 1950, 72 (6), 24182421.

6. Samsonov, G.V., Physical and Chemical Properties of Oxides: Handbook (in Russian), 1978, 110.

7. Grigoriev, I.S., Physical quantities: Handbook (in Russian), 1991, 212

8. Kimrey, H.D., Janney, M.A., Mater. Res. Soc. Symp. Proc.,1988, 124, 367-372.

9. Kishimoto, A., et al, Scripta Materialia, 2011, 64, 860-863

10. Scott, H.G., J. of Materials Science, 1975, 10, 1527-1535.

11. Baeraky, T.A., Egypt. J. Sol., 2004, 27 (1), 25 - 34.

12. D. J. Bergman, D.J., Stroud, D, Solid State Phys., 1992, 46, 147 - 269.

13. Molla, J., Preprint CEIMAT-736, 1994.

14. Yongduk, O., et al, Additive Manufacturing, 2019, 27, 586 - 594. 